\title{
Computational parametric study on efficiency of low boiling point fluid plants under heat exchange process intensification
}

\author{
Alena Likhaeva ${ }^{1}$, Sergey Grigoriev ${ }^{l}$, Evgeniy Trushin ${ }^{1}$, Marat Dasaev ${ }^{1}$ \\ ${ }^{1}$ National research university «MPEI», Moscow, Russia
}

\begin{abstract}
One of the main challenges for the energy industry is to improve the reliability and efficiency of heat exchange equipment in heating plants. Phase-change heat exchangers with low boiling point fluid (LBPF) are widely used in both conventional and renewable energy. The main objectives of increasing the efficiency of heat exchange equipment are to reduce the weight and dimensions, to increase the amount of heat transferred and to reduce the electricity consumption spent on pumping the heat transfer agent. These objectives are achieved by implementing various methods of heat exchange intensification in heat exchange equipment. A key aspect concerning application of various types of heat exchange intensifiers in heat exchange equipment is evaluation of possibility to increase their design efficiency. The paper presents the results of a computational parametric study of changes in efficiency of some LBPF-based plants when intensifying heat exchange processes by modifying functional surfaces of heat exchangers by laser ablation.
\end{abstract}

\section{Introduction}

One of the ways to save energy in power and industry is to increase the efficiency of heat exchangers. Generally, such heat exchangers are characterised by high metal consumption and considerable weight-size parameters. Intensification of heat exchange processes at condensation and boiling of fluids allows increasing thermal-hydraulic characteristics and reducing weightsize parameters of heat exchange equipment $[1,2]$.

One of the most promising ways to intensify the heat exchange processes of various fluids is based on the use of heat exchange surfaces with variable wettability (biphilic surfaces) [3-5]. In spite of the existing methods for producing biphilic surfaces [6-9], a method based on relief formation using laser equipment was proposed by the world scientific community and is being intensively developed [10, 11]

The great interest to this method is caused by the fact that during laser influence on the metal surface the texturizing of different-scale relief is performed. It is possible to control the typical geometrical parameters of the generated textures providing the change of wetting angle with sufficient accuracy due to the flexible variation of the laser emission parameters.

During condensation of heat transfer agent on biphilic surfaces, condensation is observed in different modes, film and droplet, respectively. In this way, the different surfaces have different functions in heat exchange $[8,9$, 12]: on the hydrophobic surface a condensation process takes place, intensifying the heat transfer, while the hydrophilic surface acts as a liquid transport, collecting drops from the hydrophobic surface and not allowing a condensate layer to form on it, which in turn would be an additional thermal resistance.

During boiling of working fluid in evaporator, hydrophobic areas increase the number of vapour centres due to their relief, while hydrophilic areas limit the size of bubbles formed on the hydrophobic surface, thus intensifying the heat exchange $[10,11]$.

An important aspect concerning application of various methods of heat exchange processes intensification in heat exchange equipment is evaluation of possibility to increase their design efficiency. A computational parametric study of change in efficiency was carried out for some LBPF-based plants, as follows: a heat pump system (HPS), a vapour-compression refrigeration system (VCRS) with a regenerator and an organic Rankine cycle (ORC) operating turbine. For a more complete evaluation, wide range of LBPFs was selected, whose thermal properties were sourced from the free CoolProp library [13].

\section{Research methods}

The method of calculating the efficiency of LBPF-based plants is derived from the exergy analysis technique [14]. The exergy efficiency of systems serves as an indicator of system efficiency, taking into account the thermal potential of generated energy, and is the ratio of useful exergy $E_{\text {out }}$ to input exergy $E_{\text {in }}$ (formula 1 ).

$$
\eta=\frac{E_{\text {out }}}{E_{\text {in }}}
$$


The mathematical models of the plants have been implemented using Python programming language. The calculations were performed by varying the temperature differences on the condenser and evaporator walls for a range of various LBPFs whose properties were determined using the free CoolProp library. The resulting mathematical models of the plants make it possible to evaluate how different values of temperature difference on the evaporator and condenser walls affect the exergy efficiency.

\subsection{Method of evaluation of HPS efficiency at intensification of heat exchange processes in evaporator and condenser}

Figure 1 shows a schematic diagram of HPS where the following processes are marked: 1-2 - mechanical compression, 2-3 - process of cooling and condensation of working fluid in condenser, 3-4 - process of freon aftercooling in subcooler, 4-5 - isenthalpic process of throttling in the expansion tube, 5-1 - process of freon boiling in evaporator.

The input data for the computational study is as follows:

- heat output of heat pump is $50 \mathrm{~kW}$;

- water with temperature $t_{n 1}=10^{\circ} \mathrm{C}$ at the evaporator inlet and $t_{\mathrm{H} 2}=6^{\circ} \mathrm{C}$ at the evaporator outlet is used as a source of low-temperature heat;

- water temperature at subcooler inlet $t_{s c}=35^{\circ} \mathrm{C}$ and at the condenser outlet $t_{v 2}=70^{\circ} \mathrm{C}$;

- internal relative efficiency of compressor was assumed to be $80 \%$, and electromechanical efficiency of compressor - $90 \%$.

HPS efficiency is determined by formula (2), where $q_{c}, q_{s c}$ are specific heat loads of condenser and subcooler respectively, $\mathrm{J} / \mathrm{kg} ; l_{c u}$ is specific work of compressor considering electromechanical efficiency, $\mathrm{J} / \mathrm{kg} ; \tau_{q}$ is exergy factor of heat of HPS:

$$
\eta=\frac{\left(q_{c}+q_{s c}\right) \cdot \tau_{q}}{l_{c u}}
$$

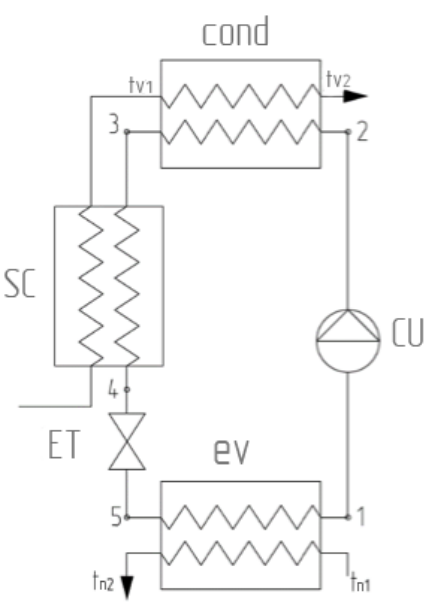

cond - condenser, CU - compressor, ev - evaporator, $\mathrm{ET}$ - expansion tube, $\mathrm{SC}$ - subcooler, $t_{v 1}, t_{v 2}$ - condenser inlet and outlet water temperatures, $t_{n 1}, t_{n 2}$ evaporator inlet and outlet water temperatures
Fig. 1. Schematic diagram of HPS

\subsection{Method of evaluation of VCRS efficiency at intensification of heat exchange processes in evaporator and condenser}

Figure 2 shows a schematic diagram of VCRS with regenerator where the following processes are marked: 1 2-mechanical compression, 2-3 - process of cooling and condensation of working fluid in condenser, 3-4 - process of freon cooling in regenerator, 4-5 - isenthalpic process of throttling in the expansion tube, 5-6- process of freon boiling in evaporator, 6-1 - afterheating of freon in regenerator.

The input data for the computational study is as follows:

- cold output is $7 \mathrm{~kW}$;

- cooled air temperature at evaporator inlet $t_{n 1}=$ $-20^{\circ} \mathrm{C}$ and at evaporator outlet $t_{n 2}=-26^{\circ} \mathrm{C}$; $25^{\circ} \mathrm{C}$

cooling water temperature at condenser inlet $t_{\mathrm{B} 1}=$

- temperature difference at the regenerator wall $20^{\circ} \mathrm{C}$;

- internal and electromechanical efficiency of compressor are $80 \%$ and $90 \%$, respectively.

VCRS efficiency is determined by formula (3), where $q_{0}$ is specific heat load of evaporator, $\mathrm{J} / \mathrm{kg} ; l_{c u}$ is specific work of compressor considering electromechanical efficiency, $\mathrm{J} / \mathrm{kg} ; \tau_{q}$ is exergy factor of cold of VCRS:

$$
\eta=\frac{q_{0} \cdot \tau_{q}}{l_{c u}}
$$

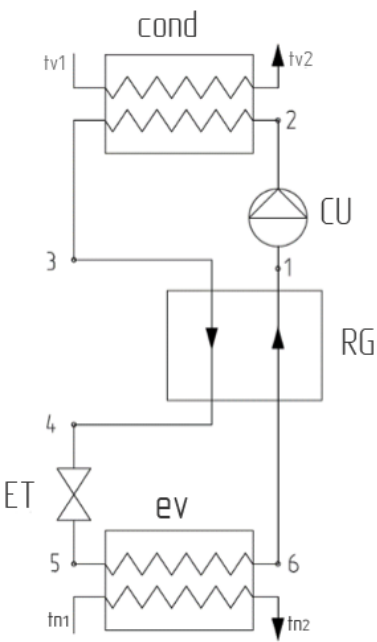

cond - condenser, CU - compressor, RG - regenerator, ev - evaporator, ET - expansion tube, $t_{v 1}, t_{v 2}{ }^{-}$ condenser inlet and outlet water temperatures, $t_{n 1}, t_{n 2}$ evaporator inlet and outlet water temperatures

Fig. 2. Schematic diagram of VCRS

\subsection{Method of evaluation of ORC efficiency at intensification of heat exchange processes in evaporator and condenser}

The schematic diagrams of the organic Rankine cycle with and without stage heater are shown in Figures 3 and 4 respectively. Figure 3 shows the following processes: 1-2 - process of freon steam expansion in turbine, 2-3 - 
cooling of freon steam in regenerator, 3-4 - process of cooling and condensation of working fluid in condenser with heat extraction by cooling water, 4-5 - process of working fluid compression in pump, 5-6 - heating of freon in regenerator, 6-1 - evaporation and superheating of steam in evaporator. Figure 4 shows the following processes: 1-2 - process of freon steam expansion in turbine, 2-3 - process of cooling and condensation of working fluid in condenser with heat extraction by cooling water, 3-4 - process of working fluid compression in pump, 4-1 - process of evaporation at constant pressure in the evaporator.

The input data for the computational study is as follows:

- secondary energy resources (SER) with temperature at evaporator inlet $t_{i 1}=100^{\circ} \mathrm{C}$ and at evaporator outlet $t_{i 2}$ $=40^{\circ} \mathrm{C}$ are used as a source of high-temperature heat;

- cooling water temperature at condenser inlet $t_{k 1}=$ $25{ }^{\circ} \mathrm{C}$ and at condenser outlet $t_{k 2}=35^{\circ} \mathrm{C}$;

- cooling water pressure drop $200 \mathrm{kPa}$;

- internal adiabatic efficiency of turbine was assumed to be $90 \%$, electromechanical efficiency of turbine $-90 \%$, and efficiency of freon pump - $70 \%$.

ORC efficiency is determined by formula (4), where $l_{\mathrm{T}}$ is specific work of turbine considering electromechanical efficiency, $\mathrm{J} / \mathrm{kg} ; q_{e}$ is specific evaporator heat capacity, $\mathrm{J} / \mathrm{kg} ; l_{f r}$ is freon pump work, $\mathrm{J} / \mathrm{kg} ; \tau_{q}$ is exergy factor of plant:

$$
\eta=\frac{l_{\mathrm{T}}}{q_{e} \cdot \tau_{q}+l_{f r}}
$$

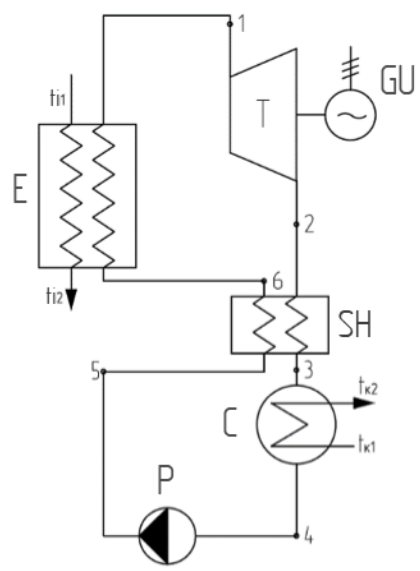

GU - generator unit, $\mathrm{C}$ - condenser, $\mathrm{E}$ - evaporator, $\mathrm{P}$ - pump, T - turbine, $\mathrm{SH}$ - stage heater, $t_{i 1}, t_{i 2}{ }^{-}$ evaporator inlet and outlet SER temperatures, $t_{\mathrm{K} 1}, t_{\mathrm{K} 2^{-}}$ condenser inlet and outlet water temperatures

Fig. 3. Schematic diagram of ORC with stage heater

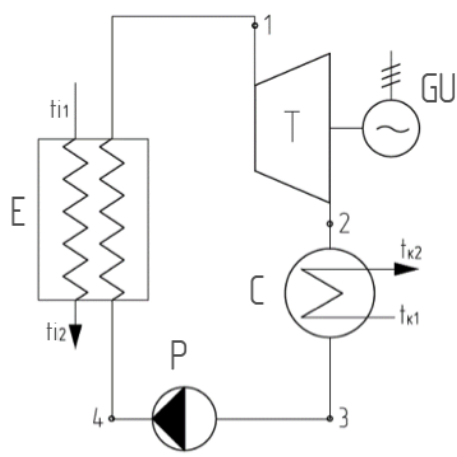

GU - generator unit, $\mathrm{C}$ - condenser, $\mathrm{E}$ - evaporator, $\mathrm{P}$ pump, $\mathrm{T}$ - turbine, $t_{i 1}, t_{i 2}$ - evaporator inlet and outlet water temperatures, $t_{\mathrm{K} 1}, t_{\mathrm{K} 2}$ - condenser inlet and outlet water temperatures

Fig. 4. Schematic diagram of ORC

\section{Results of computational parametric study and discussion}

\subsection{Results of calculation of HPS efficiency at intensification of heat exchange processes in evaporator and condenser}

The results obtained for calculation of HPS efficiency at intensification of heat exchange processes in evaporator and condenser are shown in Figure 5 as a bar chart, with working fluids on abscissa axis. However, according to the Kyoto Treaty and the Paris Agreement, some of the working fluids are eliminated from consumption [15] or can be used exclusively as raw material for other chemicals [16] (in Figures 5-9 they are indicated in grey).

Maximum efficiency of HPS as a result of heat exchange intensification corresponds to working fluids $\mathrm{R} 123, \mathrm{R} 113, \mathrm{R} 11$ and $\mathrm{R} 141 \mathrm{~b}$ and reaches the following values of efficiency and their increase of $38 \%$ and 4,15 $\%$ on average.

However, using of above-mentioned freons is limited; therefore, maximum values of efficiency and their increases out of freons being in use (for example, R1234ze(Z), R245ca, R365MFC) make on average 36,2 $\%$ and $3,03 \%$ correspondingly. In addition, the trend revealed in the analysis of results is that the average efficiency increase for the HPS evaporator is higher than for the condenser. For example, for working fluid $\mathrm{R} 1234 \mathrm{ze}(\mathrm{Z})$ value of efficiency increase in the evaporator is 2.34 times higher than in the condenser and makes $2.78 \%$. 
Thus, for heat pump system reduction of temperature difference at walls of condenser and evaporator by $5^{\circ} \mathrm{C}$ will allow to increase exergy efficiency on average by $3,99 \%$ for all working bodies and by $3,03 \%$ for working bodies not prohibited for use. refrigeration system, decrease in temperature difference on heat exchange surface of the condenser and evaporator by $5^{\circ} \mathrm{C}$ leads to increase of exergy efficiency of plants based on various LBPF on average by $8,5 \%$ (Figure 6) and by $6,5 \%$ for widely used working fluids, for example,

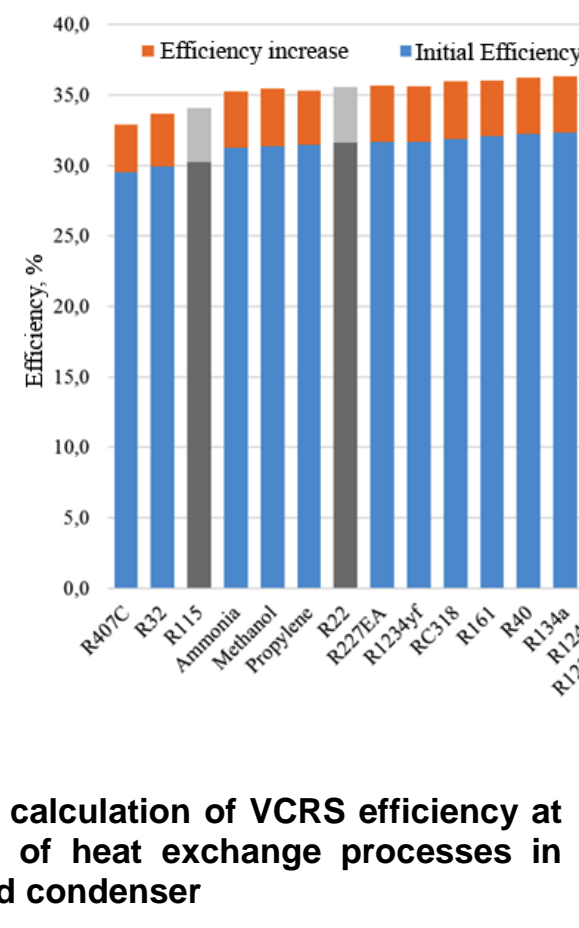

\subsection{Results of calculation of VCRS efficiency at intensification of heat exchange processes in evaporator and condenser}

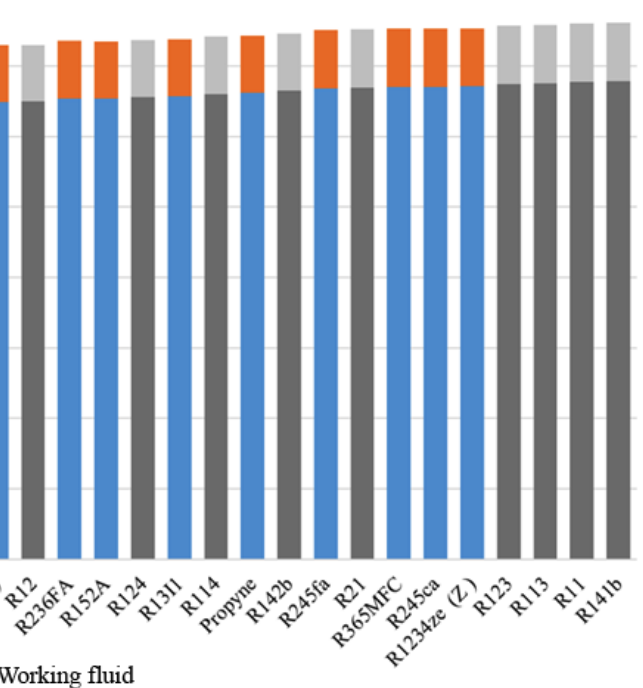

\section{$\mathrm{R} 32, \mathrm{RC} 318, \mathrm{R} 245 \mathrm{ca}$.}

It has been found that, on average, the VCRS efficiency increase in the evaporator is $13 \%$ higher than in the condenser for various LBPFs.

At intensification of heat exchange processes in the evaporator and condenser of vapour-compression

Fig. 5. Increase in HPS efficiency when varying the temperature difference on evaporator and condenser walls and depending on working agent

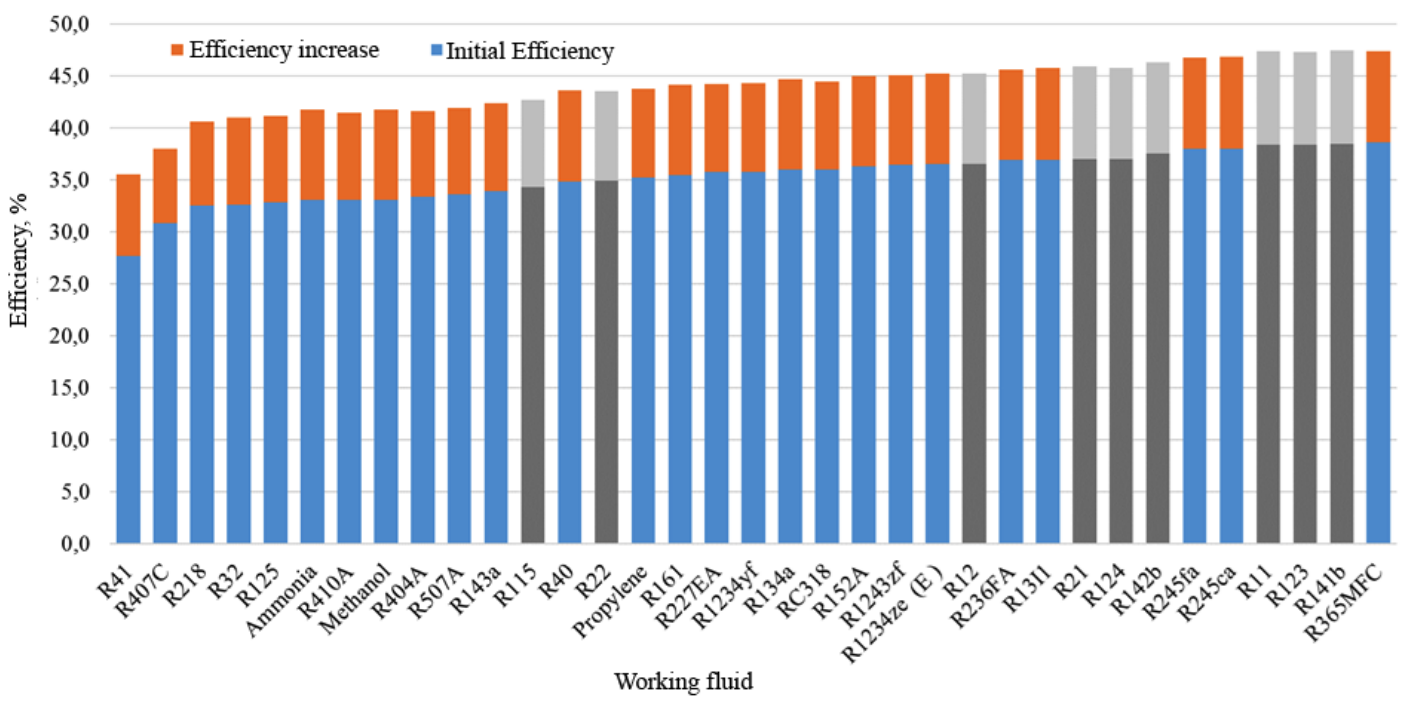

Fig. 6. Increase in VCRS efficiency when varying the temperature difference on evaporator and condenser walls and depending on working agent

\subsection{Results of calculation of ORC efficiency at intensification of heat exchange processes in evaporator and condenser}

This effect means a gradual reduction in the temperature of the subsurface near the borehole walls, which entails a reduction in the heat extracted [6].

Computational parametric study for the ORC was carried out based on two schematic diagrams (Figures 3 
and 4). Two sets of input data were used for the ORC setup without stage heater. The difference in the input data was the value of LBPF temperature after evaporator. In the first case the temperature after evaporator was in saturation area, in the second case it was in superheated steam area.

The results obtained for calculation of efficiency of simple organic Rankine cycle at intensification of heat exchange processes in evaporator and condenser are presented in Figures 7 and 8 as a bar chart. It has been found that value of both initial efficiency and efficiency increases for the system with LBPF temperature after evaporator being in saturation area for different working fluids are much higher than for the system with temperature after evaporator being in superheated steam area and are on the average $51,8 \%$ and $12,7 \%$ and 25,8 $\%$ and $7,2 \%$ respectively.

The highest efficiencies as a result of heat exchange intensification in evaporator and condenser were obtained for such working fluids as R12, R21, R11 and R142b. Average efficiency and their increase have made 56,1\% and $13,8 \%$ (Figure 7 ) and 27,6\% and 7,6\% (Figure 8). However, the above-mentioned freons are restricted in use, so working fluids currently widely used in VCRS were considered. The following values of exergy efficiencies and their increases were obtained for the case when condenser and evaporator wall temperature difference was reduced from $10^{\circ} \mathrm{C}$ to $1{ }^{\circ} \mathrm{C}$ : for $\mathrm{ORC}$ with temperature after evaporator in saturation area $55.2 \%$ and $11.35 \%$ for R1234ze(E), $54.3 \%$ and $13.53 \%$ for R13I1, $53.6 \%$ and $14.2 \%$ for Propyne; for ORC with temperature after evaporator in superheated steam area $27.5 \%$ and $7.72 \%$ for R13I1 and $26.6 \%$ and $7.7 \%$ for Propyne. Therefore, when R13I1 and Propyne are used as the working fluid, the system efficiency is the highest.

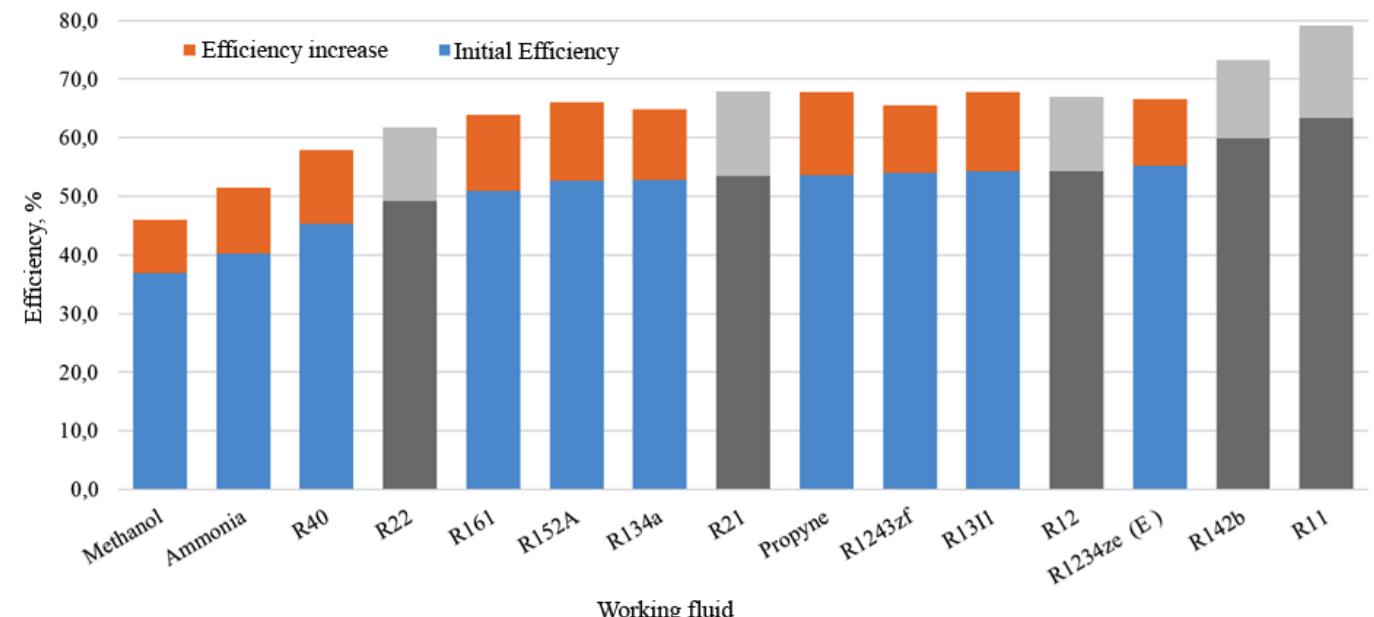

Fig. 7. Increase in ORC efficiency when varying the temperature difference on evaporator and condenser walls and depending on working agent

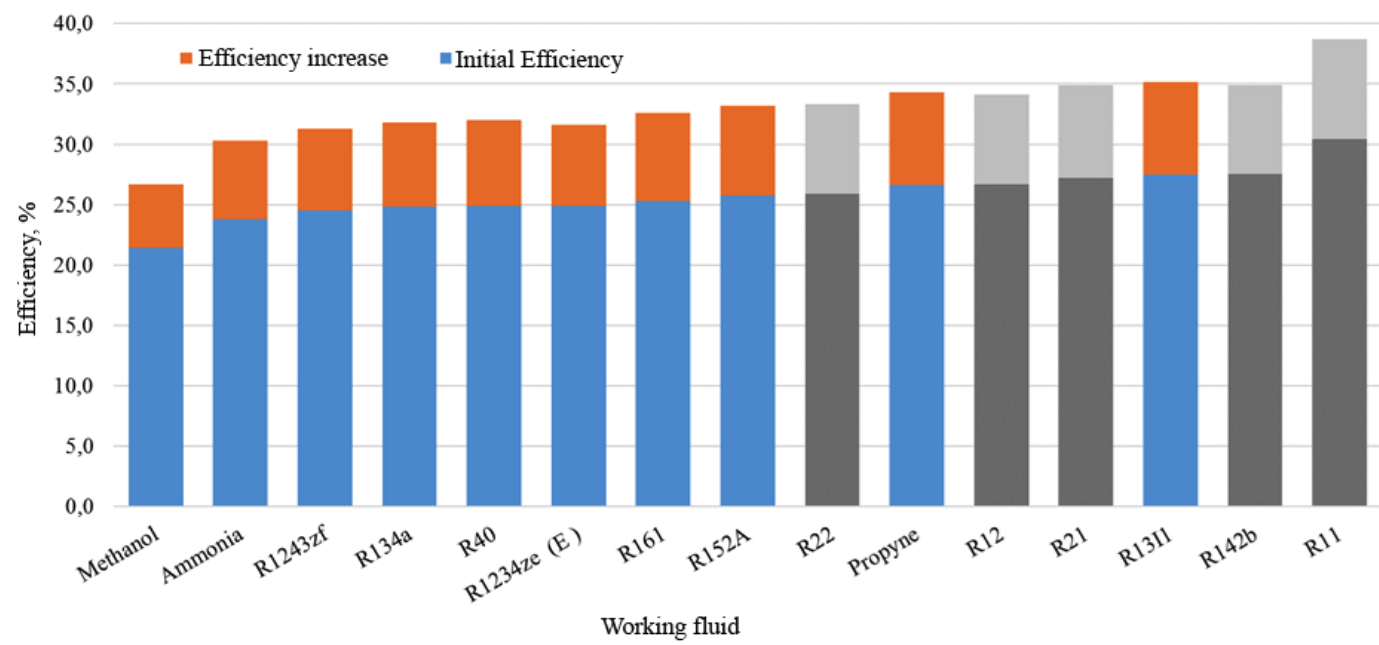

Fig 8. Increase in ORC efficiency with temperature after evaporator in superheated steam area when varying the temperature difference on evaporator and condenser walls and depending on working agent 


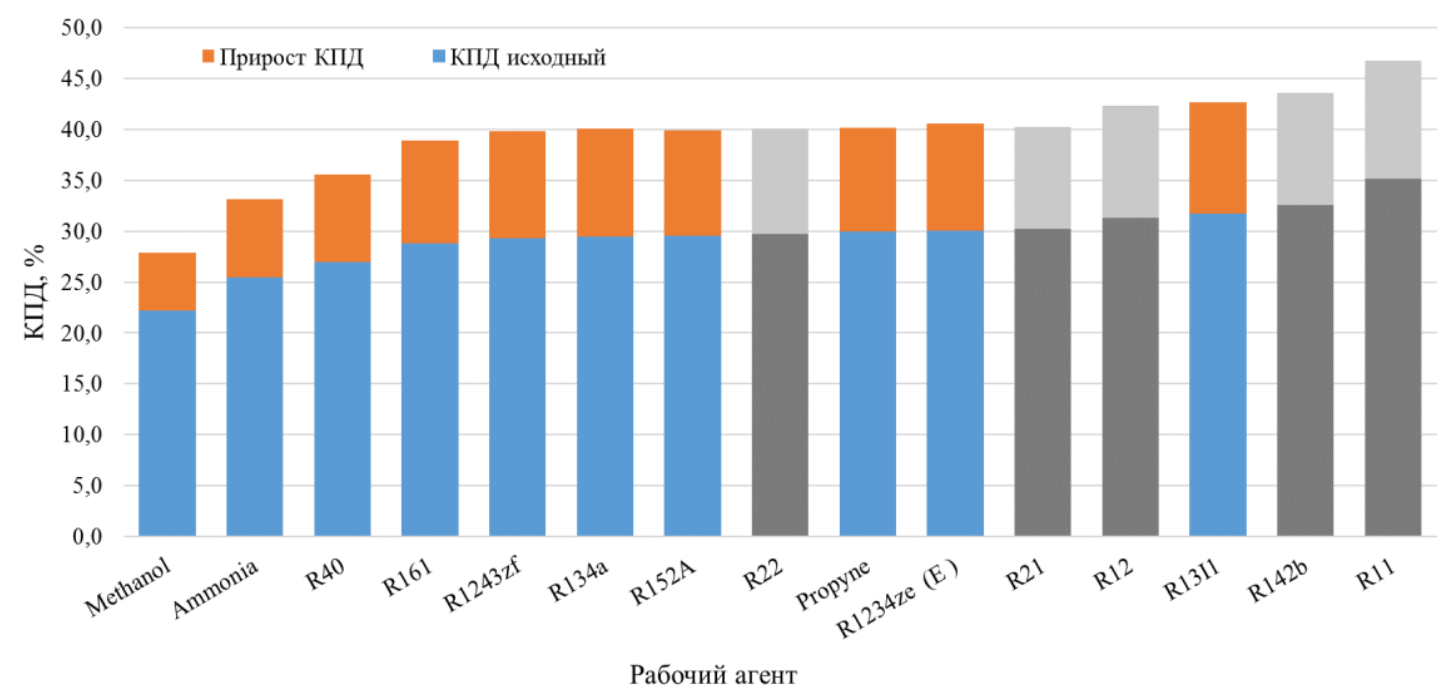

Fig.9 Increase of ORC efficiency with regenerative preheater when changing temperature difference on evaporator and condenser walls depending on working medium

When comparing the efficiency values obtained by reducing the temperature difference on condenser walls with the calculation results obtained by reducing the temperature difference on evaporator walls by the same amount, the trend was found that the average efficiency increase with modification of functional condenser surfaces is higher than with modification of functional evaporator surfaces.

Figure 9 shows the results of efficiency calculations for organic Rankine cycle with stage heater. It is noted that the highest efficiencies and their increases were obtained, as for simple Rankine cycle, for R12, R21, R11 and R142b, and the average values were $31,8 \%$ and 10,82 $\%$ correspondingly. Highest values of efficiencies for currently used working fluids as a result of heat exchange intensification in evaporator and condenser were obtained for R13I1, Propyne and R1234ze(E), making 36,6 \%, 34,7 $\%$ and $34,4 \%$ correspondingly.

The trend also remains that the average efficiency increase with modifications to the condenser functional surfaces is higher than with modifications of evaporator functional surfaces for ORC with stage heater.

As a result of computational study of organic Rankine cycle with LBPF temperature after evaporator in saturated state, in superheated steam area and organic Rankine cycle with regenerator the following average efficiency increases at reduction of temperature difference at condenser and evaporator walls from $10^{\circ} \mathrm{C}$ to $1{ }^{\circ} \mathrm{C}$ were obtained: $12.7 \%, 7.16 \%$ and $9.94 \%$ correspondingly for all working fluids and $12.18 \%, 6.93 \%$ and $9.51 \%$ correspondingly for working fluids not prohibited by the Kyoto Protocol and the Paris Agreement.

\section{Conclusions}

This effect is estimated by a conditional radius of thermal influence of the borehole, which increases over time. As

a result of the computational parametric study on efficiency of LBPF-based plants at intensification of boiling and condensation processes, the following was obtained:

- for HPS reduction of temperature difference on condenser and evaporator walls by $5{ }^{\circ} \mathrm{C}$ will allow to increase exergy efficiency depending on working fluid on average by $3,99 \%$;

- for VCRS reduction of temperature difference on condenser and evaporator walls by $5^{\circ} \mathrm{C}$ leads to $8,5 \%$ increase of exergy efficiency for different LBPF on average;

- for ORC with LBPF temperature after evaporator at saturation state, in superheated steam area and organic Rankine cycle with regenerator at $9^{\circ} \mathrm{C}$ reduction of temperature difference on condenser and evaporator walls, the increase will be $12,7 \%, 7,16 \%$ and $9,94 \%$, correspondingly.

Thus, at intensification of heat exchange processes in condenser and evaporator of LBPF-based plants an increase in efficiency of the considered heating units is achieved, which means that the way chosen to increase the efficiency of LBPF-based heat exchangers is promising.

The investigation was carried out within framework of the Project "Efficiency improvement of low boiling point fluid plants based on use of biphilic heat exchange surfaces" supported by grant from NRU «MPEI» for research programms "Energy", "Electronics, Radio Engineering and IT" and "Industry 4.0 Technologies for Industry and Robotics" in 2020-2022.

\section{References}

1. X. Quan, S. Chen, J. Li, P. Cheng; Enhanced dropwise condensation by oil infused nano-grass coatings on outer surface of a horizontal copper tube; International Communications in Heat and Mass Transfer 91 (2018), pp. 11-16.

2. Y.Q. Wang, J.L Luo, Y. Heng, D.C. Mo, S.S. Lyu; PTFE-modified porous surface: Eliminating boiling hysteresis // International Communications in Heat and Mass Transfer, Vol. 111 (2020). P. 104441. 
3. J. Yuan, Y. Wang, J. Xu, X. Ji, J. Xie Convective dropwise condensation heat transfer in mini-channels with biphilic surface // International Journal of Heat and Mass Transfer, Vol. 134 (2019). pp. 69-84.

4. P. Pontes, R. Cautela, E. Teodori, A.S. Moita, A. Georgoulas, A. Moreira. Bubble Dynamics and Heat Transfer on Biphilic Surfaces: Experiments and Numerical Simulation // Journal of Bionic Engineering, Vol. 17 (2020). pp. 809-821.

5. A.V. Ryzhenkov, A.Y. Likhaeva, S.V. Grigoryev, M.R. Dasaev, I.S. Sokolov "Ways of Intensifying the Heat Exchange Processes in a Single-Well System for Subsoil Deep Thermal Energy Pickup and Transportation" International Journal of Engineering Trends and Technology 68.11(2020):33-48.

6. N. Kofoed-Mandsberg, O. Hansen, R. Taboryski Generation of micro-droplet arrays by dip-coating of biphilic surfaces; the dependence of entrained droplet volume on withdrawal velocity // Scientific Reports (2017). Vol. 7.

7. M.M. Garimella, S. Koppu, S.S. Kadlaskar, V. Pillutla, Abhijeet, W. Choi. Difference in growth and coalescing patterns of droplets on biphilic surfaces with varying spatial distribution // Journal of Colloid and Interface Science (2017). Vol. 505. P. 1065-1073

8. J. Xie, , Q. She, , J. Xu, , C. Liang, , \& W. Li, (2020). Mixed dropwise-filmwise condensation heat transfer on biphilic surface. International Journal of Heat and Mass Transfer, Vol. 150, 119273. doi:10.1016/j.ijheatmasstransfer.2019.119273

9. K.-S. Yang, , K.-H. Lin, , C.-W. Tu, , Y.-Z. He, , \& C.-C. Wang, (2017). Experimental investigation of moist air condensation on hydrophilic, hydrophobic, superhydrophilic, and hybrid hydrophobichydrophilic surfaces. International Journal of Heat and Mass Transfer, Vol. 115, 1032-1041. doi:10.1016/j.ijheatmasstransfer.2017.08.112

10. Y. Hou, Y. Shang, M. Yu, C. Feng, H. Yu, S. Yao Tunable Water Harvesting Surfaces Consisting of Biphilic Nanoscale Topography // ACS Nano, Vol. 12, (2018). pp. 11022-11030.

11. A.V. Ryzhenkov, M.R. Dasaev, S.V. Grigoriev, A.V. Kurshakov, A.D. Karandashev, A.F. Mednikov, Experience in developing metal surfaces with controlled wettability and their efficiency estimation in installations based on low-boiling-point working fluid // International Journal of Mechanical Engineering and Technology (2018), 1240-1248.

12. Y. Shang, , Y. Hou, M. Yu, \& S. Yao, (2018). Modeling and optimization of condensation heat transfer at biphilic interface. International Journal of Heat and Mass Transfer, Vol. 122, 117-127. doi:10.1016/j.ijheatmasstransfer.2018.01.108

13. CoolProp Free Library. Access mode: http://www.coolprop.org/fluid_properties/PurePseud oPure.html

14.E.Y. Sokolov, V.M. Brodyanskiy, Energy fundamentals of heat transformation and cooling processes: Textbook for universities. - 2nd edition revised. - Moscow Energoizdat, 1981 - 320 p.

15. Guidance "Preparation for HCFC consumption reduction: main provisions relating to the use, alternatives, implications and financing for countries operating under Article 5 of the Montreal Protocol".Compiled by T. Grof, K. Murdoch. - Vienna: United Nations Industrial Development Organization (UNIDO), 2012, $240 \mathrm{p}$

16. List of products with use of ozone-depleting substances imported (exported) into Russian Federation: decree of RF Government dated December 9, 1999 No. 1368 // Official gazette. - 1999. - No. 50. - Art. 6238. 